\title{
- Analysis of Various Automated Reticulocyte Parameters in Oncology Patients
}

\author{
IJCRR \\ Section: Healthcare \\ ISI Impact Factor \\ (2019-20): 1.628 \\ IC Value (2019): 90.81 \\ SJIF (2020) $=7.893$

\section{Hemangi Joshi ${ }^{1}$, Biren Parikh ${ }^{1}$}

M.D. Pathology, Gujarat Cancer and Research Institute, Ahmedabad, Gujarat, India.

\section{ABSTRACT}

Purpose: Anaemia is quite common and yet, independent poor prognostic factor for oncology patients. Among haematological tests, reticulocyte counts are standard tests to determine different types and treatments of anaemia along with the functionality of bone marrow. Various routine and research reticulocyte parameters are generated by Mindray BC-6800. The aim and objective of this study are to analyze automated reticulocyte parameters used in routine and research in anaemic and non-anaemic oncology patients. Our hypothesis is to find whether reticulocyte parameters should be a part of a complete blood count report in oncology patients to guide clinicians for further evaluation or management.

Methods: A total of 272 routine samples were obtained randomly over three months, and run through an analyzer to evaluate reticulocyte parameters. Parameters obtained include RET\%, ARC, IRF, HFR, MFR, LFR, RetHe, RPI, MCHr and MRV.

Result: Reticulocyte parameters were generated by BC-6800 for 272 samples, from which 152 patients were anaemic while 120 patients were non-anaemic. Abnormal values for routine and research parameters like RET\%, ARC, IRF, HFR, MFR, LFR, RetHe, RPI, MCHr and MRV were 41.91\%, 59.93\%, 19.85\%, 29.04\%, 3.68\%, 27.94\%, 56.99\%, 4.78\%, 63.97\% and 55.51\%, respectively.

Conclusion: The present study showed significant abnormal results of reticulocyte parameters oncology patients. It is used for haematology laboratories to contemplate reticulocyte parameters as a part of complete blood count to guide clinicians for the management of cancer patients with the addition of interpretative comments to emphasize erythropoietic activity to increase acceptability and the efficacy of anti-cancer treatment and may also find some impact on prognosis.

Key Words: Automated reticulocyte count, Anaemia, Oncology patients

\section{INTRODUCTION}

Reticulocyte count has significant importance in the determination of the pathophysiological classification of anaemias as well as pre-recognition of resuming normal erythropoietic activity of bone marrow followed by therapeutic interventions. During haematopoietic recovery after bone marrow transplantation reticulocyte count helps in the early prediction of marrow regeneration. ${ }^{1}$ On the other hand, widespread routine use of reticulocyte count has been limited by imperfect manual microscopic counting, insufficient technical competency and a high coefficient of variations in reticulocyte counts. Enhancement in technologies of laboratory equipment and automation of cell counters helps in generating the precise and accurate results of reticulocyte count, highlighting proficiency and clinical importance of reticulocyte count enumeration. ${ }^{2}$

Fully automated methods have helped greatly in reducing turnaround time and eliminating intra-observer variability and subjectivity with more reliable statistical results as very large amounts of cells counted. ${ }^{3,4}$ In a study done by Buttarello and colleagues, the performance evaluation and comparisons of results of reticulocyte count were done by 5 automated analyzers. The result obtained was satisfactory with all methods used in automation, despite the tendency of overestimation of low reticulocyte counts. ${ }^{4}$ Automated analyzers, with the help of dyes (to bind reticulocytes RNA) and flow cytometers, proved revolutionary for reticulocyte count. These methods analyze thousands of cells and thus

\section{Corresponding Author:}

Biren Parikh, Associate Professor, Department of Oncopathology, Gujarat Cancer and Research Institute, Ahmedabad, Gujarat, India. Email: birenparikh2002@rediffmail.com; ORCID: 0000-0002-5158-9236

ISSN: 2231-2196 (Print)

Received: 30.04 .2021
ISSN: 0975-5241 (Online)

Revised: 12.05 .2021
Accepted: 26.06 .2021
Published: 01.12 .2021 
support to get objective counts. Additionally, they also reduce sampling error promoting more error-free results for low values with $\mathrm{CV}$ between $3 \%$ and $25 \%{ }^{4}$

Recognition of other automated reticulocyte parameters is an additional advantage of automated analyzers, beneficial in various clinical conditions. ${ }^{5-11}$ Mindray BC-6800 is a fully automated haematology analyzer that works on fluorescence signals of flow cytometry, and light scattering at two angles to carry out complete blood count and differential counts. ${ }^{12}$ Counting of reticulocyte is done with the help of a fluorescent dye called asymmetric cyanine adhering with cytoplasmic RNA by using a separate channel to facilitate its discrimination from matured red cells. Based on the maturity of reticulocytes, they are subdivided into three fractions by the analyzer. The proportion of the fluorescent signal intensity recognizes population at high, medium and low maturity levels. It also helps to provide some reticulocyte indices similar to those of red blood cells. ${ }^{1}$ Objective of the study is to analyze automated reticulocyte parameters used in routine and research in anaemic and non-anaemic oncology patients and based on this analysis, our hypothesis was to find whether reticulocyte parameters should be a part of a complete blood count report in oncology patients to guide clinician for further evaluation and management.

\section{MATERIALS AND METHODS}

This study presents a prospective evaluation of results of reticulocyte parameters generated by automated haematology analyzer Mindray BC-6800 at a haematology laboratory in a tertiary care oncology centre which is the National Accreditation Board for Testing and Calibration Laboratories accredited by ISO 15189 since the year 2012 .

The data have been compiled over three months from blood samples of patients who needed a complete blood count by physician's recommendations and from patients who had routine check-ups. A total of 272 samples were obtained randomly to evaluate reticulocyte parameters for this study.

Peripheral blood samples were collected in K2EDTA vacutainers and analyzed within this span of 2 hours of collection. Various automated reticulocyte parameters used in routine and research in anaemic and non-anaemic oncology patients were obtained by Mindray BC-6800. Parameters obtained include Reticulocyte percentage (RET \%), Absolute reticulocyte count (ARC), Immature reticulocyte fraction (IRF), High fluorescence reticulocyte fraction (HFR), Medium fluorescence reticulocyte fraction (MFR), Low fluorescence reticulocyte fraction (LFR), Reticulocyte haemoglobin equivalent (Ret He), Reticulocyte production index (RPI) and Mean reticulocyte haemoglobin content (Mr). Cut-offs of all parameters were assigned considering based on the existing literature. Calibration and Maintenance of the analyzer were done according to the manufacturer's recommendations. The laboratory always scored satisfactorily in external proficiency testing. Control samples were used daily to assess internal quality to achieve accurate and precise results. Further, to the evaluation of various reticulocyte parameters generated by BC-6800, Microsoft Excel version 201916.0 was used to compare the findings. To determine all these parameters of reticulocytes, cases were classified as anaemic and nonanaemic male as well as female patients.

\section{RESULTS}

A total of 272 samples was obtained. Among them, $52 \%$ of samples were of the male and $48 \%$ of females who were diagnosed as having various haematological and solid malignancies. Considering the age factor, $24 \%$ of patients were below 18 years of age and $76 \%$ of patients were more than 18 years of age. Using WHO criteria patients were classified as anaemic with haemoglobin level $<12 \mathrm{gm} / \mathrm{dl}$ for male patients and $<11 \mathrm{gm} / \mathrm{dl}$ for female patients. According to this, out of 272 patients, 152 patients were anaemic while 120 patients were non-anaemic. Among 152 anaemic patients, 64 were male and 88 were female patients. Cases were simply classified as new cases and old cases based on whether the patient has received treatment or not. All samples were run through Mindray BC-6800 automated haematology analyzer, thereafter results obtained for various reticulocytes parameters were evaluated in anaemic and non-anaemic male and female patients. Analysis of various parameters is depicted in Tables 1 and 2.

In this study, we found 114 (41.91\%) patients with abnormal RET\% values, among them $95(83.33 \%)$ patients were anaemic and 19 (16.67\%) patients were having normal haemoglobin values. Out of a total of 272 patients, 158 (58.08\%) patients were found to have normal reticulocyte counts with $89(56.33 \%)$ patients having low haemoglobin levels. A total of $163(59.93 \%)$ was found to have an abnormal ARC. Out of 272 patients, IRF, HFR and MFR values were high in 54 (19.85\%), $79(29.04 \%)$ and $10(3.67 \%)$ patients respectively. LFR was low in 76 (27.94\%) patients while 196 (72.05\%) patients were having normal limits. Abnormally low and high RHe levels were found in 155 (56.99\%) patients, while $117(43.01 \%)$ patients had normal Rhe values. Various research parameters like RPI, MCHrand MRV was found to be abnormal in 13 (4.78\%), 174 (63.97\%) and 151 (55.51\%) patients respectively. Analysis of all parameters is depicted in Tables 1 and 2.

\section{DISCUSSION}

One of the objectives of the present study was to analyze automated reticulocyte parameters in anaemic and non-anaemic 
oncology patients diagnosed with solid or haematological tumours. Among most of the haematological tests, reticulocyte counts are a standard test to determine different types and treatments of anaemia along with the functionality of bone marrow. ${ }^{13}$ Anaemia is quite common and yet substantially threatening as it may bring certain complications in oncology patients. Early detection and management may result in better clinical outcomes, signifying a better tolerance and reaction to anticancer therapy ${ }^{14}$ Cancer patients either at the advanced stage or going through anticancer therapy and recurrence of the tumour are more often reported with Anaemia. Although its occurrence may vary with cancer type, its stacks are quite high in patients suffering from haematological malignancies e.g. lymphoma, multiple myeloma. Moreover, in the list of reporting of solid tumours, lung and breast cancers find the top position, followed by malignancies of the gastrointestinal tract and gynaecology. ${ }^{15}$ Patients with colon cancer may be observed with symptoms related to anaemia signifying an indication for an occult neoplasm. ${ }^{14}$

While the effect of Anaemia can be fatal for a cancer patient, a good treatment strategy can be vital. Some studies indicate that the patients treated with cancer anaemia, especially patients with mild-to-moderate anaemia, are a key factor to finding an improved quality of life. ${ }^{16}$ Furthermore, this may help in increasing the acceptability and the efficacy of anti-cancer treatment and may also find some impact on the prognosis. ${ }^{17-19}$ Reticulocyte count aid in the discrimination of anaemias due to hypo and hyperproliferation of bone marrow and also is a noticeable marker for red blood cell production. ${ }^{10}$ A study was done by Poorana et al., wherein they took 429 pancytopenia cases and observed a considerable decreased ARC in patients with aplastic anaemia. The average decrease in ARC was found in myelodysplasia and anaemias due to nutritional deficiencies. Systemic infectious disease and Marrow infiltration were associated with extremely high ARC. ${ }^{20}$ In this study, we found significant abnormal results for RET $\%$ and ARC in random 272 patients as depicted in Table 3.

IRF is a total of HFR and MFR subdivided by analyzer based on the immaturity of reticulocytes. Numerous studies have illustrated that evaluation IRF can predict haematopoietic engraftment in allogeneic and autologous stem cell transplantation earlier than conventional markers viz. Absolute neutrophil counts and platelets. ${ }^{21,22,23}$ Apart from this, IRF also serves as an important clue in paediatric oncology patients with postchemotherapy marrow aplasia to assess marrow function. ${ }^{24}$ A study has done elsewhere by Mullier and colleagues, made out that high RET\% without uniform increased IRF in a patient with hereditary spherocytosis. ${ }^{25}$ To evaluate haemorrhage, haemolytic anaemias and aplastic anaemias, observe treatment for anaemia, erythropoietin production in patients with renal transplantation, blood transfusion demands in neonates and prematurity outcome are other applications of IRF. ${ }^{3}$ RPI value exceeding 3 correlates with therapeutical response to anaemia, recent haemorrhage and various haemolytic anaemias, while low values were associated with inefficient erythropoiesis and hyperproliferative diseases. $^{3}$

Ret $\mathrm{He}$ is a parameter about directly measuring iron levels in freshly produced reticulocytes, which may be serving as a timely signal of iron deficiency at the initial stage. A study done elsewhere revealed $\leq 27.8 \mathrm{pg} / \mathrm{L}$ cut-off point for Ret He by logistic regression analysis. In addition to this, children with lower levels of Ret He than $27.8 \mathrm{pg} / \mathrm{L}$ are at 4.5 times increased risk of iron deficiency anaemia than children with Ret He more than $27.8 \mathrm{pg} / \mathrm{L}$. Another study reported that $25 \mathrm{pg} / \mathrm{L}$ cut-off points for Ret He have $76 \%$ sensitivity and $81 \%$ of specificity. ${ }^{26} 87.6 \%$ of specificity and $65.2 \%$ sensitivity were observed in another study done on adults with iron deficiency anaemia with $<28$ pg Ret He cut-off. ${ }^{27}$ Taking into consideration all studies together, an alliance of Ret He and Iron deficiency anaemia can be illustrated. Concluding, higher haemoglobin levels correlated with higher Ret He, thus lower Ret He increases the probability of iron deficiency anaemia. Owing to the existence of reticulocytes in peripheral blood being 4 days, $\mathrm{MCHr}$ assessment is useful to detect available functional iron then. ${ }^{28,29,30}$ In a study done by Mast and colleagues evaluated the usefulness of $\mathrm{MCHr}$ to diagnose patients with iron deficiency anaemia. ${ }^{31}$ Values below 28pg for $\mathrm{MCHr}$ can predict the absence of iron stores in bone marrow with a greater negative predictive value for depleted iron than $\mathrm{MCHr}$, transferrin saturation level below $13 \%$, or ferritin level below $50 \mathrm{mg} / \mathrm{dl}$.

Studies were done for the clinical usefulness of MRV showed that patients with thalassaemia and iron deficiency anaemia had decreased MRV, but higher IRF observed in thalassaemia patients and lower reticulocyte count in iron deficiency anaemia. Further MRV along with Ret He increases during treatment of iron deficiency anaemia.-9 ${ }^{7-9}$ Patients with sickle cell anaemia receiving hydroxyurea to initiate foetal haemoglobin synthesis were related with increased reticulocytes hydration evaluated as MRV. ${ }^{32,33,34}$ Data suggests that quite a low number of requests has been received about reticulocyte parameters in our haematology department. Considering the importance of all the parameters as discussed above, we have found significant abnormal reticulocyte parameters in randomly selected oncology patients as depicted in Table 3 and Table 4. Based on this study, our hypothesis is proven that reticulocyte parameters should be an integrated part to facilitate the complete blood count reporting in oncology patients.

The following limitations of the present study should be considered: (i) The study does not include other parameters evaluated in the course of anaemia viz. Serum ferritin, serum iron, transferrin saturation etc (ii) The data accumulated is collected from a single population having a specific profile 
in a tertiary care hospital that uses a particular type of haematology analyzer. According to these factors, the results that we have shown in this accurate study may not be as accurate in other laboratories.

\section{CONCLUSION}

Anaemia is quite common and yet, independent poor prognostic factor for oncology patients. Various reticulocyte parameters have significant importance in the determination of anaemias and pre-recognition of resuming bone marrow erythropoietic activity. Recognition of routine and research automated reticulocyte parameters is an additional advantage of automated analyzers. It is of use for haematology laboratories to contemplate reticulocyte parameters as a part of complete blood count to guide clinicians for the management of cancer patients with the addition of interpretative comment to emphasize erythropoietic activity to increase acceptability and the efficacy of anti-cancer treatment and may also find some impact on the prognosis.

\section{ACKNOWLEDGEMENT}

The authors acknowledge the immense help received from the scholars whose articles are cited and included in the references of this manuscript. The authors are also grateful to the authors/editors/publishers of all those articles, journals and books from where the literature of this article has been reviewed and discussed.

\section{Abbreviation:}

RET\% - Reticulocyte percentage, ARC - Absolute reticulocyte count, IRF - Immature reticulocyte fraction, HFR - High fluorescence reticulocyte fraction, MFR - Medium fluorescence reticulocyte fraction, LFR - Low fluorescence reticulocyte fraction, Ret He - Reticulocyte haemoglobin equivalent, RPI - Reticulocyte production index, MCHr - Mean reticulocyte haemoglobin content, MRV - Mean reticulocyte volume

\section{Declarations:}

\section{Funding: Not applicable. All the required data were col- lected from routine analyzed samples.}

Conflicts of interest/Competing interests: The authors declare that they have no conflict of interest.

Consent to participate: I understand that the data collected from my participation will be used primarily for this article, and will also be used in summary form for journal publication, and I consent for it to be used in that manner.

Consent for publication: I, the undersigned, give my consent for the publication of identifiable details, which can include photographs and/or videos and/or case history and/or details within the text ("Material") to be published in the above Journal and Article.

\section{REFERENCES}

1. Buttarello M, Rauli A, Mezzapelle G. Reticulocyte count and extended reticulocyte parameters by Mindray BC-6800: Reference intervals and comparison with Sysmex XE-5000. Int J Lab Hematol. 2017;39(6):596-603.

2. Grotto HZ. Platelet and reticulocyte new parameters: why and how to use them?.Rev Bras HematolHemoter. 2016;38(4):2834.

3. Piva E, Brugnara C, Spolaore F, Plebani M. Clinical utility of reticulocyte parameters. Clin Lab Med. 2015;35(1):133-63.

4. Viana KA, Martins Filho OA, Dusse LM, Avelar RS, Avelar DM, Carvalho B, Ribeiro CM, Antonelli LR, Teixeira A, Carvalho MD. Reticulocyte count: comparison among methods. JornalBrasileiro de Patologia e Medicina Laboratorial. 2014;50(5):339-45.

5. Davis BH. Immature reticulocyte fraction (IRF): by any name, a useful clinical parameter of erythropoietic activity. Lab Hematol. 1996;2:2-8.

6. Buttarello M, Bulian P, Farina G, Petris MG, Temporin V, Toffolo L. Five fully automated methods for performing immature reticulocyte fraction: comparison in the diagnosis of bone marrow aplasia. Am J Clin Pathol. 2002;117(6):871-9.

7. Brugnara C, Laufer MR, Friedman AJ, Bridges K, Platt O. Reticulocyte haemoglobin content $(\mathrm{CHr})$ : an early indicator of iron deficiency and response to therapy. Blood. 1994;3100:3101

8. d'Onofrio G, Chirillo R, Zini G, Caenaro G, Tommasi M, Micciulli G. Simultaneous measurement of reticulocyte and red blood cell indices in healthy subjects and patients with microcytic and macrocytic anaemia. Blood. 1195;818-813

9. Brugnara C. Reticulocyte cellular indices: a new approach in the diagnosis of anaemias and monitoring of erythropoietic function. Crit Rev Clin Lab Sci. 2000;37(2):93-130.

10. Piva E, Brugnara C, Chiandetti L, Plebani M. Automated reticulocyte counting: state of the art and clinical applications in the evaluation of erythropoiesisClinChem Lab Med. 2010;48(10):1369-80.

11. Buttarello M. Laboratory diagnosis of anaemia: are the old and new red cell parameters useful in classification and treatment, how. Int J Lab Hematol. 2016:123-32.

12. Lee HT, Park PW, Seo YH, Kim KH, Seo JY, Jeong JH, Kim MJ, Ahn JY. Performance evaluation of Mindray CAL 8000 (BC-6800 and SC-120) haematology analyzer and slide maker/ stainer. J Clin Lab Anal. 2017;31(4):e22065.

13. ML N. Normocitose. reticulocitopenia. reticulócitosimaturos e eritrograma. Rev. NewsLab. 2004;63:178-90.

14. Busty F, Marchi G, Ugolini S, Castagna A, Girelli D. Anemia and iron deficiency in cancer patients: role of iron replacement therapy. Pharmaceuticals. 2018;11(4):94.

15. Grotto HZ. Anaemia of cancer: an overview of mechanisms involved in its pathogenesis. Medical oncology. 2008;25(1):12-21.

16. Cella D, Dobrez D, Glaspy J. Control of cancer-related anaemia with erythropoietic agents: a review of the evidence for improved quality of life and clinical outcomes. Ann Oncol. 2003;14(4):511-9.

17. Harper P, Littlewood T. Anaemia of cancer: impact on patient fatigue and long-term outcome. Oncology. 2005;69(Suppl. 2):2-7.

18. Calabria A, Katz A. Management of anaemia in cancer patients. Future Oncol.. 2011(4):507-17. 
19. Littlewood TJ, Bajetta E, Nortier JW, Vercammen E, Rapoport B, Epoetin Alfa Study Group. Effects of epoetin-alfa on hematologic parameters and quality of life in cancer patients receiving nonplatinum chemotherapy: results of a randomized, doubleblind, placebo-controlled trial. J ClinOncol. 2001;19(11):286574.

20. PooranaPriya P, Subhashree AR. Role of absolute reticulocyte count in the evaluation of pancytopenia-a hospital-based study. $\mathrm{J}$ Clin Diagn Res. 2014;8(8): FC01.

21. Noronha JF, De Souza CA, Vigorito AC, Aranha FJ, Zulli R, Miranda EC, Grotto HZ. Immature reticulocytes as an early predictor of engraftment in autologous and allogeneic bone marrow transplantation. Clin Lab Haematol. 2003;25(1):47-54.

22. Torres A, Sánchez J, Lakomsky D, Serrano J, Alvarez MA, Martín C, Valls C, Nevado L, Rodriguez A, Casaño J, Martínez F. Assessment of hematologic progenitor engraftment by complete reticulocyte maturation parameters after autologous and allogeneic hematopoietic stem cell transplantation. haematologica. 2001;86(1):24-9.

23. Molina JR, Sanchez-Garcia J, Torres A, Alvarez MA, Serrano J, Casaño J, Gomez P, Martinez F, Rodriguez A, Martin C. Reticulocyte maturation parameters are reliable early predictors of hematopoietic engraftment after allogeneic stem cell transplantation. Biol Blood Marrow Transplant. 2007;13(2):172-82.

24. Luczyński W, Ratomski K, Wysocka J, Krawczuk-Rybak M, Jankiewicz P. Immature reticulocyte fraction (IRF)-an universal marker of hemopoiesis in children with cancer?.Adv Med Sci. 2006;51:188-90.

25. Mullier F, Lainey E, Fenneteau O, Da Costa L, Schillinger F, Bailly N, Cornet Y, Chatelain C, Done JM, Chatelain B. Additional erythrocytic and reticulocyte parameters helpful for diag- nosis of hereditary spherocytosis: results of a multicentre study. Ann Hematol. 2011;90(7):759-68.

26. Canals C, Remacha AF, Sarda MP, Piazuelo JM, Royo MT, Romero MA. Clinical utility of the new Sysmex XE 2100 parameter-reticulocyte haemoglobin equivalent in the diagnosis of anaemia. Haematologica. 2005;90(8):1133-4.

27. Rungngu SL, Wahani A, Mantik MF. Reticulocyte haemoglobin equivalent for diagnosing iron deficiency anaemia in children. PaediatrIndones. 2016;56(2):90-4.

28. Brugnara C. A hematologic "gold standard" for iron-deficient states?.Clin Chem. 2002;981-982

29. Brugnara C. Iron deficiency and erythropoiesis: new diagnostic approaches. Clin Chem. 2003;49(10):1573-8.

30. Brugnara C, Adamson J, Auerbach M, Kane R, Macdougall I, Mast A. Iron deficiency: what are the future trends in diagnostics and therapeutics?.Clin Chem. 2013;59(5):740-5.

31. Mast AE, Blinder MA, Lu Q, Flax S, Dietzen DJ. Clinical utility of the reticulocyte haemoglobin content in the diagnosis of iron deficiency. Blood. 2002;99(4):1489-91.

32. Brugnara $\mathrm{C}$. Use of reticulocyte cellular indices in the diagnosis and treatment of haematological disorders. Int J Clin Lab Res. 1998;28(1):1-1.

33. De Franceschi L. Pathophysiology of sickle cell disease and new drugs for the treatment. Mediterr J Hematol Infect Dis. 2009; 1(1)

34. Quarmyne MO, Risinger M, Linkugel A, Frazier A, Joiner C. Volume regulation and $\mathrm{KCl}$ cotransport in reticulocyte populations of sickle and normal red blood cells. Blood Cells Mol Dis. 2011;47(2):95-9. 